\title{
Local probe of vortex pinning energies in the Bose glass
}

\author{
K. M. Beauchamp, L. Radzihovsky, E. Shung, and T. F. Rosenbaum \\ The James Franck Institute and Department of Physics, The University of Chicago, Chicago, Illinois 60637 \\ U. Welp and G. W. Crabtree \\ Materials Science Division, Argonne National Laboratory, Argonne, Illinois 60439
}

(Received 5 July 1995)

\begin{abstract}
Columnar defects provide strong pinning centers for vortices in high- $T_{c}$ superconductors, increasing global critical currents. Using a magnetometer array of micron dimensions, we characterize the local field profiles in untwinned single crystals of $\mathrm{YBa}_{2} \mathrm{Cu}_{3} \mathrm{O}_{7-\delta}$ with equivalent columnar defect densities $B_{\phi}$. We find that the critical current is large only where the internal magnetic field $B<B_{\phi}$. In regions of the crystal where $B>B_{\phi}$, the critical current is sharply reduced. We model both local and global critical current measurements by generalizing the Bean picture to the case of irradiated high- $T_{c}$ superconductors.
\end{abstract}

Many applications of the cuprate superconductors depend on their ability to carry high currents with zero resistance in the presence of a magnetic field. Unfortunately, critical currents in wires, films, and crystals are generally low due to the motion of magnetic vortices. Columnar defects, a form of correlated disorder, provide strong pinning centers for vortices along their entire length. These defects have been found to reduce vortex motion, thereby increasing critical currents and expanding the region in temperature and field in which the superconductors have zero resistance. ${ }^{1-3}$ Columnar defects have the potential to be an important technological tool in the fabrication of high-current-density wire; thus, it is necessary that the field dependence of the critical current density be understood in detail.

High-temperature superconductors are routinely characterized using instruments such as commercial superconducting quantum interference device (SQUID) magnetometers, which provide a global average over a sample's volume. Such measurements indicate that the critical current $J_{c}$ decreases gradually with applied magnetic field $H$ in crystals with columnar defects. By contrast, we demonstrate here that there is an acute spatial variation in $J_{c}$ at fixed $H$. A linear array of photolithographically defined Hall probe magnetometers reveals that the critical current is large only in regions of the crystal where there are fewer vortices than columnar defects $\left(B<B_{\phi}\right)$ and that, where there are more vortices than columnar defects $\left(B>B_{\phi}\right), J_{c}$ falls precipitously. At low temperatures, the vortices are believed to freeze into a "Bose glass," with fluxoids localized on a disordered array of columnar defects. ${ }^{4-7}$ We attribute the spatial variation in critical current to a crossover from a strongly pinned Bose glass $^{4,5}$ for internal magnetic fields $B<B_{\phi}$ to a weakly pinned Bose glass for internal fields $B>B_{\phi} .{ }^{8}$ By modifying the conventional Bean model ${ }^{9}$ to include a critical current which changes abruptly at $B_{\phi}$, we are able to model both local and global $J_{c}$ measurements.

We have measured the internal magnetic field of untwinned $\mathrm{YBa}_{2} \mathrm{Cu}_{3} \mathrm{O}_{7-\delta}$ single crystals with and without columnar defects at $T=5 \mathrm{~K}$ as a function of applied field $H$ and of distance $x$ from the edge of the crystal. The Bi thin- film Hall magnetometers ${ }^{10}$ have active areas of $5 \mu \mathrm{m}$ $\times 10 \mu \mathrm{m}$. The crystals are polyhedral platelets of area in the $a-b$ plane greater than $150 \mu \mathrm{m} \times 150 \mu \mathrm{m}$ and thickness less than $20 \mu \mathrm{m}$. Columnar defects were introduced along the $c$ axis by irradiation with $605-\mathrm{MeV} \mathrm{Xe}$ ions at the ATLAS source; they extend from face to face of the crystal. These defects are randomly positioned with an average density of $B_{\phi} / \phi_{0}$, where $B_{\phi}$ is the field at which all of the defects are filled by vortices and $\phi_{0}=2.07 \times 10^{-7} \mathrm{G} / \mathrm{cm}^{2}$ is the flux quantum associated with a vortex. We have studied crystals without defects and with $B_{\phi}=2,10$, and $20 \mathrm{kG}$.

The critical currents, which arise from a balance between the local Lorentz force on flux lines due to the local supercurrents and the pinning force, traditionally have been extracted from global magnetization hysteresis loops via the Bean method. ${ }^{9}$ Using Maxwell equations to relate local current to $B$ field gradient gives the Bean equation which determines the internal field profile in terms of the critical current $J_{c}$,

$$
\vec{\nabla} \times \vec{B}=\frac{4 \pi}{c} \vec{J}_{c} .
$$

In a slab-shaped sample which has its long dimension along the direction of the field, the critical current is given by

$$
J_{c y}=\frac{c}{4 \pi} \frac{d B_{z}}{d x}
$$

it can be measured directly, in principle, from the global magnetization hysteresis. ${ }^{9}$ Although the Bean model assumes a field-independent critical current, in this geometry the Bean method also has been used to determine $J_{c}(H){ }^{1,2}$

Most critical current measurements in high- $T_{c}$ crystals, however, are made with the magnetic field applied perpendicular to the plane of the crystal (platelet geometry). In this configuration, demagnetization effects cause a bending of the field lines, resulting in a critical current which is not simply proportional to the gradient of the field. Then, the perpendicular component of the internal magnetic field near the edge of the sample, where the curvature of the field lines is strongest, is a nonlinear function of distance and the Bean method cannot be used directly to determine critical 

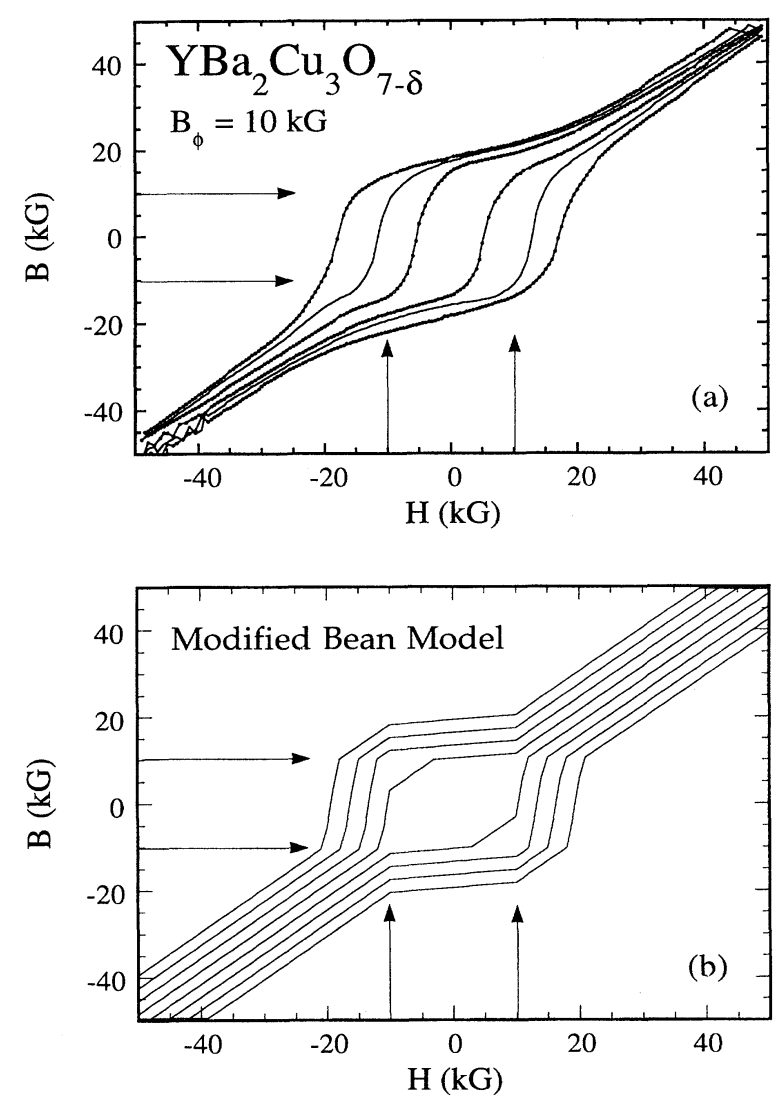

FIG. 1. (a) Local internal magnetic field $B$ vs applied field $H$ at $25 \mu \mathrm{m}$ (inner curve), $50 \mu \mathrm{m}$, and $70 \mu \mathrm{m}$ (outer curve) distances from the crystal edge at $T=5 \mathrm{~K}$. (b) Calculated local hysteresis loops using modified Bean model with $B_{\phi}=10 \mathrm{kG}$.

currents. ${ }^{11,12}$ Expressions for global virgin magnetization ${ }^{12}$ (measured from zero internal magnetic field) or global virgin remanent magnetization ${ }^{11}$ for the platelet geometry can be used to determine a field-independent critical current, but cannot be used to determine $J_{c}(H)$. We have measured the low field $\left(B<B_{\phi}\right)$ critical current at $T=5 \mathrm{~K}$ using the global virgin magnetization method and found $J_{c 1}=(7 \pm 0.8) \times 10^{6}$ $\mathrm{A} / \mathrm{cm}^{2}$ for $B_{\phi}=10 \mathrm{kG}, J_{c 1}=(3.3 \pm 0.3) \times 10^{6} \mathrm{~A} / \mathrm{cm}^{2}$ for $B_{\phi}=2 \mathrm{kG}$, and $J_{c}=(6.3 \pm 0.5) \times 10^{6} \mathrm{~A} / \mathrm{cm}^{2}$ for no columnar defects. The critical current for the unirradiated crystal is a strong function of temperature, and it falls below the values for crystals with columnar defects at higher $T$.

Despite flux line bending in the platelet geometry, near the center of the sample the magnetic field is nearly linear in distance and $d B / d x$ scales with critical current. Hence, a local measurement of the internal magnetic field can provide an accurate relative determination of $J_{c}(H)$. Using a Hall probe array, we have explored the variation in internal magnetic field with applied magnetic field at different distances $x$ from the edge of the crystal. We plot in Fig. 1(a) local hysteresis loops at three positions near the center of the 150 $\mu \mathrm{m} \times 180 \mu \mathrm{m}, B_{\phi}=10 \mathrm{kG}$ crystal: at $x=25,50$, and 70 $\mu \mathrm{m}$. Demagnetization effects are evident for the probe closest to the edge of the crystal, but become less important farther from the edge, where the field gradient is nearly lin-

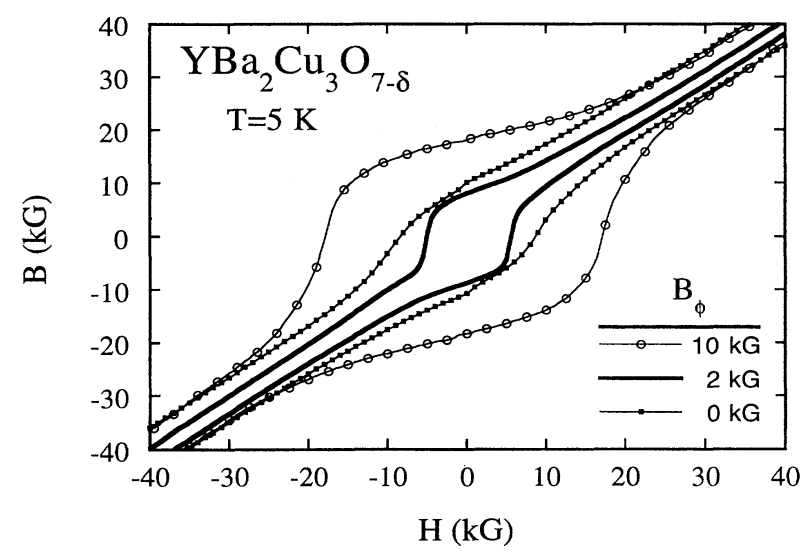

FIG. 2. Hysteresis loops at $T=5 \mathrm{~K}$ near the centers of crystals with no columnar defects, $B_{\phi}=2 \mathrm{kG}$, and $B_{\phi}=10 \mathrm{kG}$.

ear. Each probe displays three distinct values of $d B / d H$, independent of $x$. The slope $d B / d H=1$ at large $|H|$, as is expected from the conventional Bean model. However, at small $|H|$ and $|B|$, both $d B / d H \ll 1$ and $d B / d H / \gg 1$ slopes appear. The crossovers between these slopes occur near $|B|=B_{\phi}$, also independent of the Hall probe position.

We find similar behavior in crystals with $B_{\phi}=2$ and 20 $\mathrm{kG}$, with the width of the hysteresis loop at a given distance from the edge of the crystal increasing with increasing $B_{\phi}$, indicating an increase in critical current with increasing $B_{\phi}$. We compare in Fig. 2 local hysteresis loops taken near the centers of crystals with $B_{\phi}=0,2$, and $10 \mathrm{kG}$, respectively. The crystal without columnar defects has a hysteresis width and a $d B / d H$ which changes only slightly with applied field, indicating a small change in $J_{c}$ with $H$. Consistent with the global virgin magnetization measurement, the local measurement indicates that the crystal with no defects has a higher critical current at $T=5 \mathrm{~K}$ than the crystal with $B_{\phi}=2 \mathrm{kG}$ and that $J_{c}$ increases with $B_{\phi}$ in the subset of crystals with columnar defects.

We can use the local nature of our probe to determine the dependence of $J_{c}$ on $H$. We plot in Fig. 3(a) the internal magnetic field $B$ vs position $x$ at a variety of applied magnetic fields on the increasing branch of the hysteresis loop for the $B_{\phi}=10 \mathrm{kG}$ crystal. Again, we restrict our measurements to a region near the center of the crystal in order to minimize demagnetization effects. We clearly see small $d B /$ $d x$, and thus small $J_{c}$, for $|B|>B_{\phi}$, and large $d B / d x$, and thus large $J_{c}$, for $|B|<B_{\phi}$. We find corresponding results for the $B_{\phi}=2 \mathrm{kG}$ crystal, whereas the field gradient was nearly constant over the whole field range for the unirradiated crystals. ${ }^{13}$ This method gives us a ratio of the critical current for $|B|<B_{\phi}$ to that for $|B|>B_{\phi}$ of $10.8 \pm 0.8$ for $B_{\phi}=10 \mathrm{kG}$ and $7.4 \pm 0.7$ for $B_{\phi}=2 \mathrm{kG}$. Hence, we find that columnar defects induce in the same crystal, in the same applied magnetic field, different critical currents in regions where there are fewer vortices than defects and where there are fewer defects than vortices. The spatial profile $B(x)$ obtained via our local Hall probe highlights vividly the inadequacy of using the conventional Bean model to determine bulk critical currents.

A critical current which changes sharply at $B_{\phi}$ can arise in 

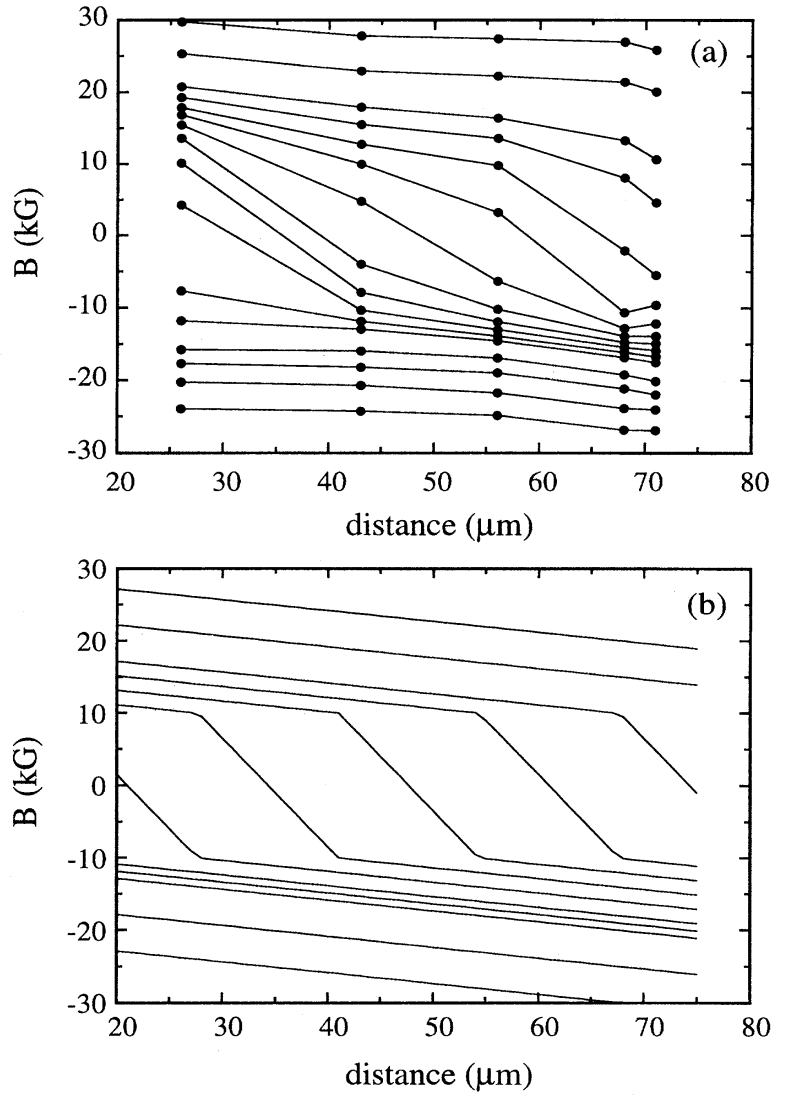

FIG. 3. (a) Position dependence $x$ of internal magnetic field $B$ at a variety of applied fields $H$ for the $B_{\phi}=10 \mathrm{kG}$ crystal at $T=5 \mathrm{~K}$. The critical current $(\propto d B / d x)$ depends strongly on field, plummeting above $B_{\phi}$. (b) $B$ vs $x$ for a series of external fields in the modified Bean model.

a Bose glass superconductor because the presence of columnar defects leads to an additional pinning energy scale distinct from the vortex interaction energy scale. ${ }^{1,8}$ For $B<B_{\phi}$, there are a sufficient number of columnar pins for each flux line to be localized on a defect and to be able to take advantage of the strong pinning it provides. The pinning force acts over the length of the columnar defect and is characterized by the pinning energy per unit length, $U_{0}$, which is of order $\left(\phi_{0} / 4 \pi \lambda\right)^{2}$, where $\lambda$ is the London penetration length. It is this strong pinning which leads to a significant shift in the irreversibility line first observed by Civale et al. ${ }^{1}$ We refer to this regime of $B$ field as the strongly pinned Bose glass (SBG), discussed in detail by Nelson and Vinokur. ${ }^{4,5}$

We argue that there exists a distinct regime when $B>B_{\phi}$. Energetic considerations now favor the occupation of the interstices between the vortices already strongly pinned by the columnar defects. At very low temperatures, these interstitial vortices also will be localized, but in this case by repulsion from the vortices on the columnar defects. Since the randomly localized columnar vortices will appear to the interstitial flux lines as a random, $z$-independent potential, the interstitial vortices should be described as well by Bose glass physics. ${ }^{5}$ However, the localization energy scale of the interstitial vortices $U_{i} \ll U_{0}$ due to the repulsion from neighboring vortices strongly localized on columnar defects. This leads to a (model-dependent) estimate that $U_{i} \sim \varepsilon_{l} / d n^{1 / 2} \ll U_{0}$, where $\varepsilon_{l}=\gamma \varepsilon_{0} \ln (\lambda / \xi), \gamma$ is the material anisotropy factor, $d$ is the spacing between columnar defects, and $n$ is the flux line areal density. ${ }^{5}$ There follows a reduced $J_{c}\left(B>B_{\phi}\right)=c U_{i} / \phi_{0} d \ll J_{c}\left(B<B_{\phi}\right)$. We expect that the equilibrium properties of this phase will be similar to that of the SBG in all respects except for the reduced pinning energy scale which determines a reduced $J_{c}$ and a $T_{\mathrm{BG}}(B)$. We note that the observed shift of the irreversibility line to higher temperature when columnar defects are introduced ${ }^{1}$ supports the idea of two distinct pinning energy scales. The increase in $T_{\mathrm{BG}}$ reflects a transition from processes governed by the vortex interaction energy (controlling the melting-freezing transition line in the defect-free sample) to those dominated by the higher pinning energy from columnar defects.

Based on the experimental discovery of two different slopes, $d|B| / d x$, in Fig. 3(a), together with the theoretical considerations outlined above, we propose a modified Bean model appropriate to the description of a superconductor with columnar defects. The model is a simple generalization of the original Bean model to include a critical current which changes sharply as a function of $B$ at $B_{\phi}$. Our modified Bean model is defined simply as a Bean model with the conditions that for $|B|<B_{\phi}, J_{c}=J_{c 1}$, and for $|B|>B_{\phi}$, $J_{c}=J_{c 2}$, where $J_{c 1}>J_{c 2}$. We do not rule out field dependence of $J_{c}$ for each regime; however, we stress that the change in $J_{c}$ at $B_{\phi}$ is much more pronounced than that within one regime. $J_{c 1}$ and $J_{c 2}$ are to be understood as the components of an effective, but not literal, two-fluid model. As illustrated in Fig. 3(b), this modified Bean model reproduces all the main features observed in the data of Fig. 3(a). Additionally, averaging the internal magnetic field over the entire crystal would give a global magnetization (and an associated critical current) which appears to decrease monotonically with increasing applied field.

The modified Bean model also predicts significantly more complicated hysteresis loops [Fig. 1(b)] than in the unirradiated superconductors, with features that are independent of the placement of the local probe. There are break points, indicated by arrows, which occur at $H=B_{\phi}$ and at $B=B_{\phi}$, the coordinates for which are given in Table I. Furthermore, while the segments $d B / d H=1$ for $|B|>B_{\phi}$ as in a conventional hysteresis loop, for $|B|<B_{\phi}$ the steep slope is the ratio $J_{c 1} / J_{c 2}$ and the shallow slope is $J_{c 2} / J_{c 1}$. In the limit of negligible demagnetization corrections, hysteresis measurements could serve as an additional means to extract $J_{c 1} / J_{c 2}$.

The precipitous drop in $J_{c}$ at $|B|=B_{\phi}$ revealed by our magnetometer array cannot be accounted for by a gradual crossover in the functional form of $J_{c}(H)$. Rather, we believe that it is a manifestation of the two distinct pinning energies, $U_{0}$ and $U_{i}$. A separation of pinning energy scales for $B>B_{\phi}$ leads to a significant number of new experimental predictions for Bose glass superconductors. ${ }^{8}$ For $B>B_{\phi}$, the qualitative features can be obtained with an extended twofluid model of two species of vortices, those that are strongly localized on columnar pins of fraction $B_{\phi} / B$ and the remaining $1-B_{\phi} / B$ fraction of weakly localized, interstitial vortices. 
TABLE I. Modified Bean model parameters for superconductors with columnar defects [see Fig. 1(b)].

$(H, B)$ break points in the local hysteresis loop:

Increasing branch for $x<2 B_{\phi} / J_{c 1}$ :

$\left(-B_{\phi},-B_{\phi}-J_{c 2} \cdot x\right), \quad\left(-B_{\phi}+J_{c 1} \cdot x,-B_{\phi}\right)$,

$\left(B_{\phi}, B_{\phi}-J_{c 1} \cdot x\right)$

$\left(B_{\phi}+J_{c 2} \cdot x, B_{\phi}\right)$

Decreasing branch for $x<2 B_{\phi} / J_{c 1}$ :

$\left(B_{\phi}, B_{\phi}+J_{c 2} \cdot x\right), \quad\left(B_{\phi}-J_{c 1} \cdot x,-B_{\phi}\right)$,

$\left(-B_{\phi},-B_{\phi}+J_{c 1} \cdot x\right)$,

$\left(-B_{\phi}-J_{c 2} \cdot x,-B_{\phi}\right)$

Increasing branch for $x>2 B_{\phi} / J_{c 1}$ :

$\left(-B_{\phi},-B_{\phi}-J_{c 2} \cdot x\right), \quad\left(B_{\phi},-B_{\phi}\left(1-2 J_{c 2} / J_{c 1}\right)-J_{c 2} \cdot x\right)$,

$\left(B_{\phi}\left(1-2 J_{c 2} / J_{c 1}\right)+J_{c 2} \cdot x,-B_{\phi}\right)$

$\left(B_{\phi}+J_{c 2} \cdot x, B_{\phi}\right)$

Decreasing branch for $x>2 B_{\phi} / J_{c 1}$ :

$\underline{\left(B_{\phi}, B_{\phi}+J_{c 2} \cdot x\right), \quad\left(-B_{\phi}, B_{\phi}\left(1-2 J_{c 2} / J_{c 1}\right)+J_{c 2} \cdot x\right), \quad\left(-B_{\phi}\left(1-2 J_{c 2} / J_{c 1}\right)-J_{c 2} \cdot x, B_{\phi}\right), \quad\left(-B_{\phi}-J_{c 2} \cdot x,-B_{\phi}\right)}$

Our observation that the critical current decreases abruptly at $B_{\phi}$ has important ramifications for applications where vortex pinning is necessary over wide field ranges and may mean that a uniform density of columnar defects has a limited usefulness in this type of application. The maximum density of columnar defects which can be used without degrading the superconducting properties must be determined for practical viability and will provide an upper limit on the fields the materials will be able to withstand. Alternatively, clever engineering of columnar defect density may allow for high critical currents over a wide range of magnetic fields. A quantitative understanding of the dependence of $J_{c}$ on $B$ and $T$ in irradiated high- $T_{c}$ superconductors will be a necessary precondition.

We thank V. Vinokur for illuminating discussions, and we are indebted to J. Hettinger and B. Glagola for making the crystal irradiation at ATLAS a success. This work was supported by the National Science Foundation (DMR91-20000) through the Science and Technology Center for Superconductivity. G.W.C. acknowledges support from the U.S. Department of Energy, Basic Energy Sciences-Materials Science under Contract No. W-31-109-ENG-38.
${ }^{1}$ L. Civale et al., Phys. Rev. Lett. 67, 648 (1991).

${ }^{2}$ W. Gerhauser et al., Phys. Rev. Lett. 68, 879 (1992).

${ }^{3}$ M. Konczykowski, V. M. Vinokur, F. Rullier-Albenque, Y. Yeshurun, and F. Holtzberg, Phys. Rev. B 47, 5531 (1993).

${ }^{4}$ D. R. Nelson and V. M. Vinokur, Phys. Rev. Lett. 68, 2398 (1992).

${ }^{5}$ D. R. Nelson and V. M. Vinokur, Phys. Rev. B 48, 13060 (1993).

${ }^{6}$ W. Jiang et al., Phys. Rev. Lett. 72, 550 (1994).

${ }^{7}$ R. C. Budhani, W. L. Holstein, and M. Suenaga, Phys. Rev. Lett.
72, 566 (1994).

${ }^{8}$ L. Radzihovsky, Phys. Rev. Lett. 74, 4923 (1995).

${ }^{9}$ C. P. Bean, Phys. Rev. Lett. 8, 250 (1962).

${ }^{10}$ G. T. Seidler et al., Phys. Rev. Lett. 70, 2814 (1993).

${ }^{11}$ E. H. Brandt and M. Indenbom, Phys. Rev. B 48, 12893 (1993).

${ }^{12}$ E. Zeldov, J. R. Clem, M. McElfresh, and M. Darwin, Phys. Rev. B 49, 9802 (1994).

${ }^{13}$ The small dimensions of the $B_{\phi}=20 \mathrm{kG}$ crystal prevented an accurate measurement of $d B / d x$. 